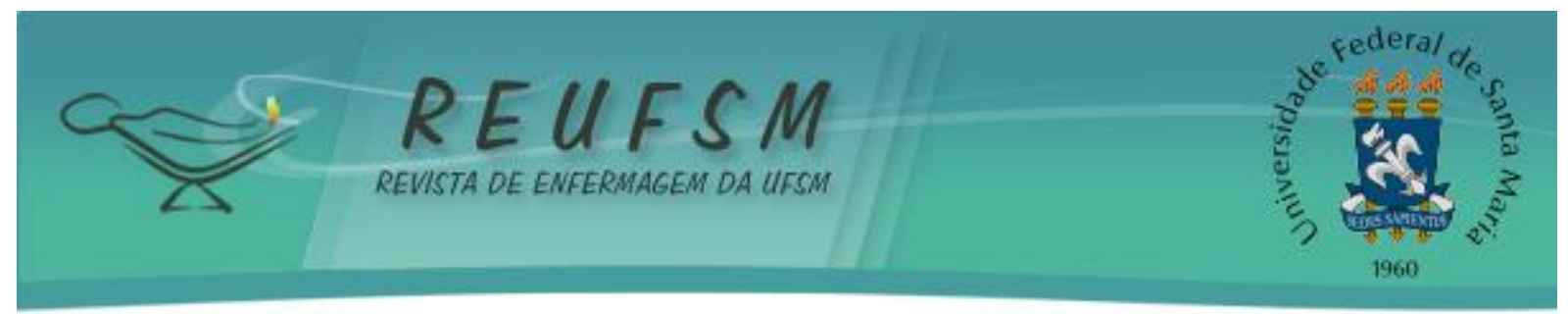

ARTIGO ORIGINAL

\title{
AVALIAÇÃO DA FERRAMENTA WEB NANDA: DIAGNÓSTICO DE ENFERMAGEM COM ESTUDANTES DE ENFERMAGEM
}

\author{
ASSESSMENT OF THE WEB TOOL NANDA.ES FOR NURSING DIAGNOSES BY NURSING \\ STUDENTS
}

\section{EVALUACIÓN DE LA HERRAMIENTA WEB NANDA.ES DE DIAGNÓSTICOS DE ENFERMERÍA CON ESTUDIANTES DE ENFERMERÍA}

Doi: $10.5902 / 2179769210713$

\author{
Francisca Sánchez Ayllón ${ }^{1}$ \\ Adriana Catarina de Souza Oliveira ${ }^{2}$ \\ Maravillas Giménez Fernández ${ }^{3}$ \\ Juan Antonio Flores Martín ${ }^{4}$ \\ Isabel Morales Moreno ${ }^{5}$ \\ Paloma Echevarría Pérez ${ }^{6}$
}

RESUMO: Objetivos: avaliar a percepção de ajuda que os alunos de $2^{\circ}$ ano de Enfermagem da Universidade Católica de Murcia tem através da "ferramenta Nanda.es", para a realização de planos de cuidado. Método: estudo descritivo, transversal com a participação de 109 estudantes de enfermagem do segundo ano, os dados foram analisados com o software SPSS, versão 19. Resultados: $73,4 \%$ dos alunos acreditam que a ferramenta apoiava bastante ou muito, para $87,5 \%$ e $90,3 \%$ respondeu que a ferramenta web thes apoiava para uma melhor compreensão da metodologia de enfermagem, $33 \%$ que a dificuldade para realizar um plano de cuidados é nada ou pouco difícil: $88,9 \%$ recomendaria aos profissionais enfermeiros, e 91,7\% recomendaria aos estudantes de enfermagem. Conclusões: esta ferramenta web é um bom aliado para implementar diagnósticos de enfermagem e planos de cuidado, com boa precisão e otimização de tempo.

Descritores: Enfermagem; Tecnologia educacional; Assistência de enfermagem; Diagnóstico de enfermagem.

ABSTRACT: Aims: evaluate the perception of help from students of the 2nd year of Nursing at Catholic University of Murcia concerning "Nanda.es Tool" for carrying out care plans. Methods: Descriptive, transverse study involving 109 nursing students in the second year, the data were analyzed with SPSS software, version 19.Results: $73.4 \%$ of students believe the tool helps a lot or very much, $87.5 \%$ and $90.3 \%$ responded that the web tool

\footnotetext{
1 Profesora de la facultad de Enfermería de la Universidad Católica de Murcia (UCAM). Doctoranda del programa de Investigación en Ciencias Sociosanitaria de la UCAM. Máster en Investigación en Ciencias Sociosanitarias. Universidad Católica San Antonio de Murcia. España. Correo: fsayllon@ucam.edu

2 Profesora de la facultad de Enfermería de la Universidad Católica de Murcia (UCAM). Doctora en Salud Pública. Universidad Católica San Antonio de Murcia. España. Correo: acatarina@ucam.edu.

3 Profesora de la facultad de Enfermería de la Universidad Católica de Murcia (UCAM). Doctora en Ciencias Sociales y de la Salud. Universidad Católica San Antonio de Murcia. España. Correo: mgimenez@ucam.edu.

${ }^{4}$ Enfermero. Máster en Investigación Sociosanitaria. Hospital Universitario Los Arcos del Mar Menor. San Javier. Servicio Murciano de Salud. Correo jafloresmartin@gmail.com

${ }^{5}$ Profesora de la facultad de Enfermería de la Universidad Católica de Murcia (UCAM). Doctora en Ciencias Sociales y de la Salud. Universidad Católica San Antonio de Murcia. España. Correo: imorales@ucam.edu.

${ }^{6}$ Profesora de la facultad de Enfermería de la Universidad Católica de Murcia (UCAM). Doctora.en Antropología Social y Cultural Universidad Católica San Antonio de Murcia. España. Correo: pechevarría@ucam.edu.
} 


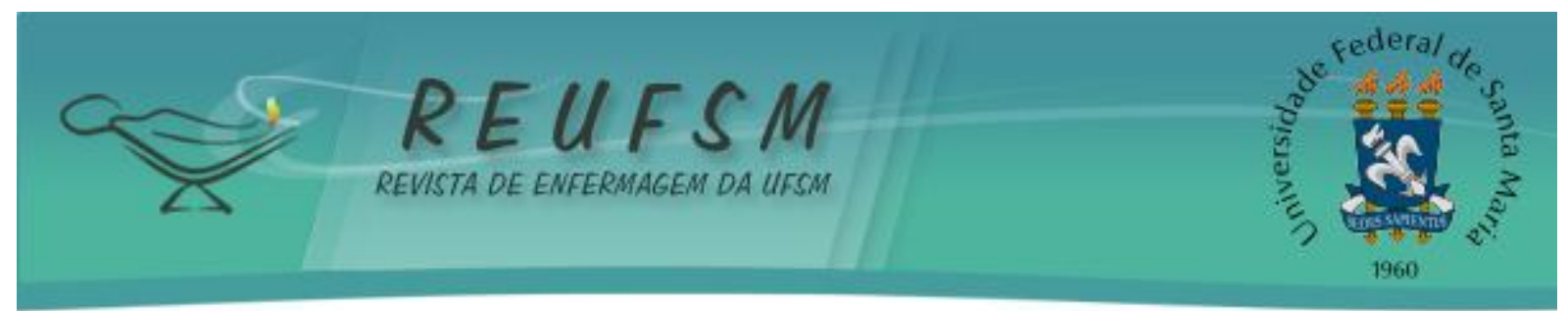

supports them for better methodological understanding of nursing; 33\% believe that the difficulty in making a care plan is not difficult or a little difficult; $88.9 \%$ would recommend it to nurses, and $91.7 \%$ would recommend it to nursing students. Conclusions: This web tool is a good ally for nursing diagnoses and care plans, with good accuracy and time improvement.

Descriptors: Nursing; Educational technology; Nursing care; Nursing diagnosis.

RESUMEN: Objetivos: evaluar la percepción de ayuda que los alumnos de $2^{\circ}$ Grado Enfermería de la Universidad Católica de Murcia tienen a través de la "Herramienta Nanda.es" para la realización de planes de cuidados. Método: estudio descriptivo, transversal con la participación de 109 estudiantes de enfermería $2^{\circ}$ Grado. Los datos fueron analizados con el software SPSS, versión 19. Resultados: el 73,4\% de los alumnos creen que la herramienta web les apoya para una mejor comprensión metodológica de enfermería; el 33\% opinan que la dificultad para realizar un plan de cuidados es nada o poco difícil; el $88.9 \%$ se lo recomendaría las enfermeras; y $91.7 \%$ se le recomendaría a los estudiantes de enfermería. Conclusiones: esta herramienta web es un buen aliado para implementar diagnósticos de enfermería y planes de cuidados, con una buena precisión y mejora del tiempo.

Descriptores: Enfermería; Tecnología educacional; Cuidados de enfermería; Diagnósticos de enfermería.

\section{INTRODUCCIÓN}

Según NANDA International, un diagnóstico de enfermería es un juicio clínico sobre la respuesta de un individuo, familia o comunidad, frente a procesos vitales / problemas de salud reales o potenciales. Los diagnósticos enfermeros proporcionan la base para la selección de intervenciones enfermeras destinadas a lograr los objetivos de los que la enfermera es responsable. ${ }^{1}$ La formulación de los diagnósticos de enfermería requiere de los estudiantes una adecuada comprensión de la enfermería, de las ciencias sociales y humanas, así como una serie de competencias relativas a la evaluación de la salud, la clínica, el razonamiento y la capacidad de integrar los conocimientos y conectarlos unos con otros. ${ }^{2}$

El proceso enfermero es la aplicación del método científico en la práctica asistencial de la enfermería, donde el diagnóstico enfermero es una etapa básica. ${ }^{3} \mathrm{La}$ evolución de la metodología enfermera en los últimos años ha sido positiva pues se ha incrementado el número de diagnósticos de enfermería, así como su calidad. Igualmente, ha aumentado la incidencia de diagnósticos en el ámbito de lo biológico que en el psicológico, que son menos habituales. ${ }^{3-4}$ Una de las áreas que a los estudiantes de enfermería les resultan más difíciles cuando se están formando en los procesos enfermeros, es la determinación del apropiado diagnóstico enfermero a partir de los datos recogidos de un individuo o paciente. Sabemos que los estudiantes de enfermería que se gradúan sin haber aprendido a trabajar con las taxonomías para la realización de diagnósticos y que no han entendido la importancia de trabajar este proceso tienden a no usar los diagnósticos enfermeros cuando se gradúan y son resistentes a su uso. ${ }^{4}$

Algunos investigadores han analizado las dificultades en el aprendizaje del proceso diagnóstico en estudiantes de enfermería. Echevarría y colaboradores realizaron un estudio con los objetivos de detectar los dominios y clases de mayor y de menor dificultad en el aprendizaje de la taxonomía NANDA en la población de usuarios registrados en la página web www.nanda.es. ${ }^{5}$ 


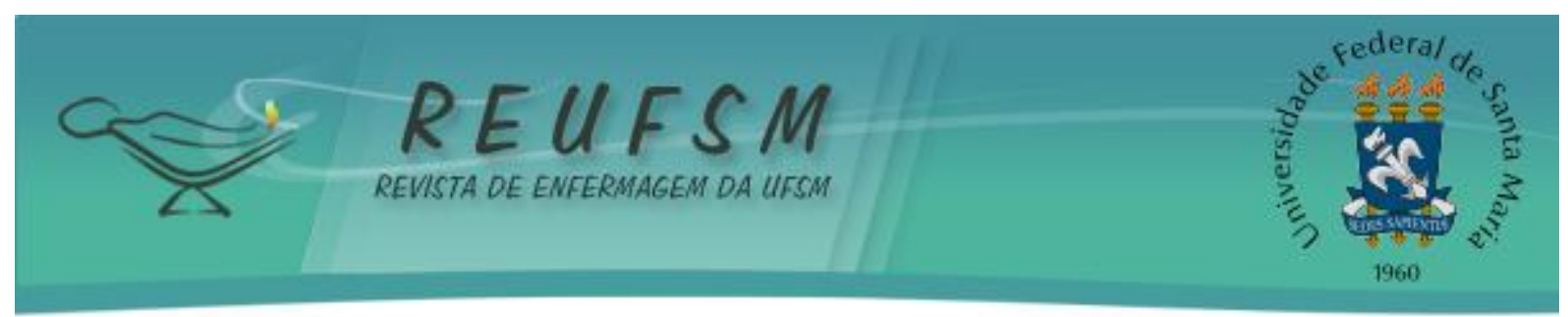

Los dominios con más aciertos fueron: eliminación e intercambio y sexualidad, y las clases correspondientes sistema gastrointestinal y función sexual. Los dominios y clases que mayor dificultad suponen para los usuarios de la taxonomía NANDA son Autopercepción, Actividad/Reposo, Afrontamiento/Tolerancia al estrés y Principios Vitales, con sus correspondientes clases Autoconcepto, Actividad/Ejercicio, Respuesta de afrontamiento y Congruencia de las acciones con los valores/creencias. Respecto a las etiquetas diagnósticas con mayor proporción de fallo, se encuentran los siguientes: disposición para mejorar la energía (00187), riesgo de soledad (00054), retraso en la recuperación quirúrgica (00100), riesgo de comportamiento de salud (00188) e incumplimiento del tratamiento (00079). ${ }^{5}$

Los estudiantes utilizan más diagnósticos basados en evidencias de tipo físico que diagnósticos basados en la valoración de evidencias psicológicas, ${ }^{4}$ lo que muestra la mayor dificultad de valoración de éstas últimas, por lo que, en el ámbito profesional, los diagnósticos enfermeros derivados de problemas de autonomía o de carácter psicológico, como ansiedad, temor o afrontamiento ineficaz, apenas son motivo de registro. ${ }^{6} \mathrm{La}$ interpretación de los datos procedentes de la valoración de los pacientes es compleja y puede dar lugar a imprecisiones en el diagnóstico enfermero. ${ }^{7}$ De hecho, sólo una tercera parte de los estudiantes se sienten preparados para realizar una valoración y un diagnóstico enfermero. ${ }^{4}$ La valoración es la primera etapa del Proceso de Atención de Enfermería (PAE). En ella se realiza la valoración del estado de salud de la persona, familia o comunidad y tiene como propósito recoger y organizar información. Cuando la recogida de datos se organiza de forma exhaustiva, se facilita la evaluación de la salud y el diagnóstico enfermero. ${ }^{8}$ El desarrollo de habilidades de pensamiento crítico es muy importante para mejorar la precisión del diagnóstico enfermero, ${ }^{9}$ aunque se trata de un proceso complejo y difícil de enseñar. ${ }^{10}$

Lunney identifica seis habilidades cognitivas y diez hábitos mentales como dimensiones del pensamiento crítico en el proceso diagnóstico; las habilidades cognitivas son: capacidad de análisis, aplicación de estándares, capacidad de discriminación, búsqueda de información, predicción y transformación de la información; los hábitos mentales son: confianza, visión del contexto, creatividad, flexibilidad, curiosidad, integridad intelectual, intuición, apertura de mente, perseverancia y reflexión. ${ }^{7}$

El uso adecuado de las habilidades de pensamiento crítico es un soporte para la validez de los diagnósticos hechos a partir de los datos. Los principales problemas identificados en el proceso de valoración, son el tiempo empleado y la dificultad para valorar determinadas necesidades, como la necesidad de creencias y valores, la necesidad de ocuparse y la necesidad de evitar peligros. ${ }^{8}$

La adquisición de conocimientos sobre taxonomía diagnóstica puede ser favorecida por el uso de programas de formación y herramientas on-line. ${ }^{11-13} \mathrm{Ha}$ sido aplicado un programa de enseñanza consistente en un proceso de resolución de problemas de nueve etapas con el objetivo de mejorar la implementación de planes de cuidados. ${ }^{2}$ Las dificultades para la implementación del Proceso enfermero, no sólo se relaciona con la práctica profesional, sino también en la práctica académica. ${ }^{14}$ Los resultados indican una mejora en la realización de planes de cuidados tras la aplicación del programa de resolución de problemas. Sin embargo, se constata la necesidad de realizar un esfuerzo formativo con el fin de mentalizar a los estudiantes de la importancia de efectuar diagnósticos enfermeros utilizando un lenguaje estandarizado. Se realizó un estudio con el objetivo de proporcionar una guía de procedimiento paso a paso para la elaboración de un diagnóstico de enfermería que cumpla con los criterios necesarios para su inclusión en la NANDA International y los sistemas de clasificación $N N N .^{1}$ Una guía de procedimiento 


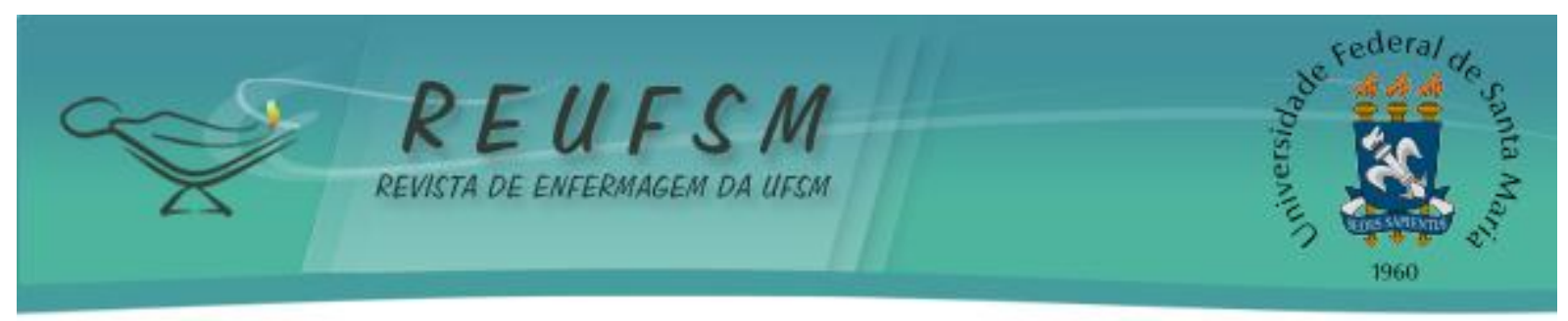

ayudará a las enfermeras a la implementación de un plan de cuidados y mejorará la calidad de los diagnósticos. ${ }^{1}$

La formación de grupos de trabajo con el objetivo de mejorar la organización de los cuidados en las unidades de hospitalización y desarrollar un método de trabajo en enfermería que permita la elaboración de planes de cuidados estandarizados y el uso de las taxonomías enfermeras ha dado resultados satisfactorios. ${ }^{15} \mathrm{El}$ uso de taxonomías contribuye a homogeneizar el lenguaje y la disciplina de los cuidados reduciendo la variabilidad en la práctica asistencial, determinando el nivel adecuado de los resultados esperados y estableciendo líneas de actuación para la continuidad de cuidados. ${ }^{15}$

Para facilitar el manejo de esta taxonomía a los estudiantes de enfermería, se diseñó, por un equipo de investigadores de la Universidad Católica San Antonio (Murcia, España), una herramienta informática denominada "Herramienta Web nanda.es" cuyo objetivo es la implementación de un algoritmo en la realización del plan de cuidados que relaciona los códigos de los diagnósticos enfermeros enlazándolos con los criterios de resultados y vinculando éstos a unas intervenciones determinadas. Como se indica en la propia página web en la que se aloja la "Herramienta Webnanda.es" (www.nanda.es), nanda.es es una herramienta de libre acceso, gratuita y sin ánimo de lucro para la difusión de la Metodología Enfermera, diseñada para facilitar el aprendizaje de las Taxonomías y facilitar la toma de decisiones del profesional o estudiante de enfermería a la hora de diseñar un plan de cuidados.

La herramienta Nanda.es como metodología de enseñanza y aprendizaje ¿genera una percepción de ayuda a los estudiantes de enfermería a la hora de elaborar los planes de cuidado y un mejor grado de aprendizaje del Proceso de Atención de Enfermería? Por eso, hemos planteado como objetivos evaluar la percepción de ayuda que los alumnos de $2^{\circ}$ Grado de Enfermería de la UCAM tienen a través de la "Herramienta Nanda.es" para la realización de planes de cuidados, detectar la percepción de dificultades de la "Herramienta Nanda.es" para la realización de planes de cuidados, detectar las posibles modificaciones de la "Herramienta Nanda.es" para su mejora en la realización de planes de cuidado, conocer el grado de uso y evaluar la herramienta web Nanda.es, un instrumento de aprendizaje para la implementación de diagnósticos de enfermería y planes de cuidados.

\section{METODO}

Este estudio descriptivo transversal se desarrolló en la Universidad Católica San Antonio (UCAM) de Murcia (España). En él participaron los estudiantes de $2^{\circ}$ Grado de Enfermería que se encontraban matriculados en el curso académico 2009-2010. La población estaba formada por 212 sujetos distribuidos en cinco grupos ( $a, b, c, d$ y e). De ellos, 195 sujetos cumplieron los criterios de inclusión: estar matriculado en el curso 20092010 en $2^{\circ}$ curso del grado de Enfermería de la Universidad Católica San Antonio; haber cursado en el primer semestre la asignatura de metodología de enfermería (este criterio es cumplido por 195 sujetos pertenecientes a los grupos a, b y e); el criterio de exclusión es no estar registrado como usuario de la herramienta Web Nanda.es. La recogida de datos se realizó en la primera semana de mayo de 2010, seleccionando las fechas para cada grupo en función de la estimación de mayor afluencia de alumnos. Para eso se estudiaron los horarios de cada grupo con el fin de determinar la fecha exacta. La muestra fue seleccionada siguiendo un procedimiento de muestreo no probabilístico consecutivo, mediante la aplicación del cuestionario a todos los alumnos que se encontraban presentes en las aulas en la fecha elegida. Los alumnos fueron informados del procedimiento que se estaba realizando y se solicitó la colaboración de todos ellos, con la indicación de que si 


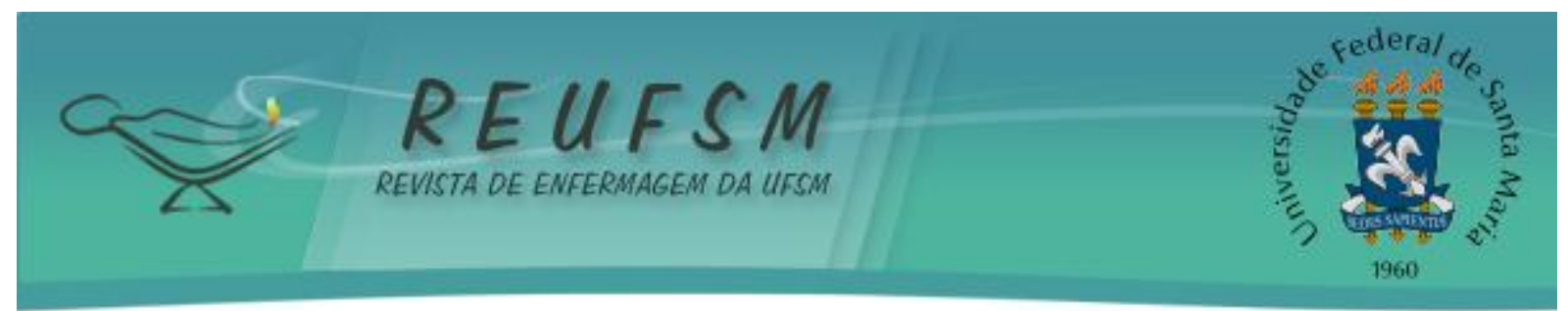

algún alumno no deseaba rellenar el cuestionario, podía abandonar el aula. Fueron informados, siguiendo la ley de protección de datos, del tratamiento que se iba a dar a la información recogida, que va a ser totalmente confidencial, ya que el cuestionario era anónimo. Finalmente, contestaron el cuestionario 109 sujetos. El estudio fue aprobado por el Comité de Ética e Investigación de la Universidad Católica de Murcia.

Para la recogida de datos el equipo investigador elaboró un cuestionario que recopila información en cinco aspectos relativos al uso de la "Herramienta web e-NANDA". La primera sección hace referencia a la percepción de ayuda que tienen los usuarios de la Herramienta. Está compuesta por ocho ítems que evalúan la percepción de ayuda en una escala tipo Likert, con puntuaciones desde uno (nada de ayuda) hasta cinco (mucha ayuda). La máxima puntuación en la escala de percepción de ayuda es, 40 puntos, que indicaría que la Herramienta es de mucha ayuda. La mínima puntuación es de ocho, que indicaría nada de ayuda. En la segunda sección evalúa la percepción de dificultades de los usuarios en el uso de la Herramienta. Para ello hemos elaborado siete ítems en una escala tipo Likert, desde uno (ninguna dificultad) hasta cinco (mucha dificultad), relativos a los sucesivos pasos en la elaboración del Plan de Cuidados. La máxima puntuación en la escala, 35 puntos, indicaría que la Herramienta es muy difícil. La mínima puntuación, siete, indicaría que la Herramienta no tiene dificultad alguna en su uso. La tercera sección se ha elaborado con la finalidad de detectar el grado de uso de la Herramienta, a través de 6 ítems que evalúan el uso de diferentes secciones mediante una escala dicotómica (sí, no). La cuarta sección del cuestionario está formada por tres ítems, mediante una escala dicotómica (sí, no), con el objetivo de identificar las secciones sugeridas a modificar por los usuarios. La quinta sección es la valoración global de la Herramienta Web Nanda.es, compuesta por cuatro ítems dicotómicos (sí, no) que evalúa el ajuste a las expectativas del usuario sobre el diagnóstico enfermero seleccionado, evalúa las expectativas esperadas sobre el plan de cuidados, la recomendación a otros posibles usuarios y la ayuda para comprender la asignatura, y un último ítem con una escala de uno a diez que evalúa la puntuación global que el usuario de da a la herramienta. Finalmente, se propuso, un apartado en el que se recogen las observaciones y comentarios de los sujetos. Los datos fueron analizados con el software SPSS, versión 19. En las pruebas de los Coeficientes de Correlación de Pearson se ha utilizado un $a=0.05$. Para estudiar la asociación que existe entre la valoración global de la Herramienta Web Nanda.es con la percepción de ayuda y análisis de la percepción de dificultad, se ha utilizado el análisis de las correlaciones de Pearson. La fiabilidad de las diferentes secciones arroja un resultado satisfactorio en todas ellas, con valores del coeficiente alfa de Cronbach de 0.87 para la escala de Percepción de ayuda y de 0.96 para la escala de Detección de dificultades.

\section{RESULTADOS E DISCUSSAO}

Para los 109 estudiantes que contestaron el cuestionario, a una amplia mayoría $(66,9 \%)$ les parece que la Herramienta les proporciona bastante o mucha ayuda para conocer mejor las Taxonomías y sólo un $11,9 \%$ creen que les sirve de poco o nada para este conocimiento. También a la gran mayoría de alumnos $(73,4 \%)$, indican que la Herramienta les proporciona bastante o mucha ayuda, en la realización de planes de cuidados y sólo el $8,5 \%$ creen que les ayuda poco o nada. Por último, la Herramienta en general es de bastante o mucha ayuda para el $65,1 \%$ de los alumnos, siendo únicamente el $14,6 \%$ de los alumnos el que piensa que le ayuda poco o nada.

La percepción de ayuda media general, en una escala de 1 a 5 , donde 1 es "nada" de ayuda y 5 es "mucha" ayuda, ha sido de 3,48 (desviación típica $=0,85$ ). Para un 33\%, la 


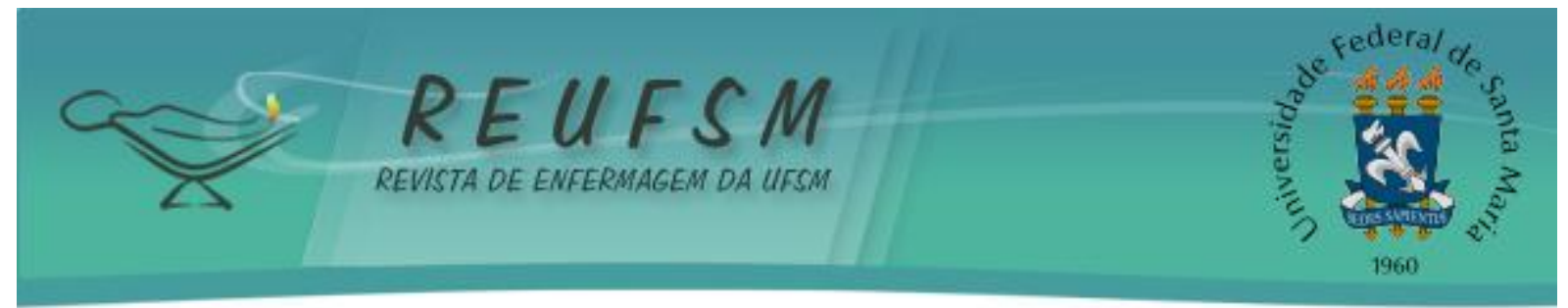

realización de un plan de cuidados con la Herramienta, fue nada o poco difícil, de dificultad media para el $27,5 \%$ y bastante o muy difícil para el $39,5 \%$ de los alumnos.

La percepción media de dificultad medida por la escala ha sido de 2,93 (desviación típica $=1,05)$, donde 1 es nada difícil y 5 muy difícil.

En cuanto AL análisis del grado de uso, Un $89,4 \%$ de los alumnos han utilizado el Juego NANDA, el $24,8 \%$ han accedido a la sección de noticias, el $77,1 \%$ han accedido a la sección Mis estadísticas/Mis planes y el 54,9\% han entrado en la sección Top 20/Estadísticas usuarios.

En cuanto a las modificaciones que los alumnos realizarían, el $26.6 \%(n=25)$ considera que habría que modificar la forma de seleccionar las taxonomías, el $18.5 \%$ ( $\mathrm{n}=$ 17) cree que habría que realizar una modificación del Juego Nanda y el $21.6 \%(n=21)$ opina que habría que modificar la presentación final del Plan de Cuidados.

La valoración global media de la Herramienta fue de $7.29(S D=1.4)$ sobre un máximo de 10. En general, la valoración general de la Herramienta es bastante positiva, como indican los altos porcentajes de alumnos que afirman que los diagnósticos eran los esperados y que ayuda a comprender la asignatura de Metodología Enfermera (tabla 1). En este sentido, cumple con las expectativas de un alto porcentaje de alumnos, que recomendarían mayoritariamente esta Herramienta tanto a las enfermeras asistenciales como a otros estudiantes (tabla 1). Los resultados de la valoración realizada por los estudiantes de enfermería de la Herramienta Web Nanda.es muestran, en general, que se trata de un instrumento satisfactorio en el desarrollo de aprendizaje de los procesos de atención en enfermería, específicamente en la aplicación de la taxonomía NANDA.

Tabla 1- Frecuencias y porcentajes de la valoración general de la Herramienta Web Nanda.es

\begin{tabular}{lc}
\hline Valoración general & $\mathrm{n}(\%)$ \\
Los diagnósticos eran los esperados (sí) & $81(84.4)$ \\
La Herramienta Web cumple las expectativas (sí) & $84(87.5)$ \\
Recomendarías a una enfermera asistencial (sí) & $90(90.0)$ \\
Recomendarías a otros alumnos (sí) & $97(93.3)$ \\
\hline
\end{tabular}

Los aspectos relacionados con la valoración global indican que los diagnósticos emitidos a través del algoritmo implementado en la Herramienta Web son, en la mayoría de los casos los esperados, cumpliendo, por lo tanto, las expectativas de los usuarios. No obstante, no se ha comparado la precisión y eficiencia con un procedimiento tradicional, por lo que no se puede afirmar que la Herramienta Web sea superior al procedimiento tradicional a la hora de realizar los diagnósticos. Un estudio sobre la precisión y eficiencia de un sistema computerizado de diagnóstico enfermero no pudo confirmar que los diagnósticos realizados mediante el nuevo sistema eran más precisos que los realizados utilizando el manual, aunque sí se observó una mayor eficiencia en el tiempo empleado para llegar a los mismos diagnósticos. ${ }^{16}$

Una de las claves de la satisfacción de los estudiantes con la Herramienta Web parece residir en la posibilidad de obtener retroalimentación de los diagnósticos anteriormente realizados y de la información almacenada en la sección Mis estadísticas/Mis planes, que es la más relacionada con la valoración global positiva, siendo la segunda más utilizada tras la sección Juego Nanda. 


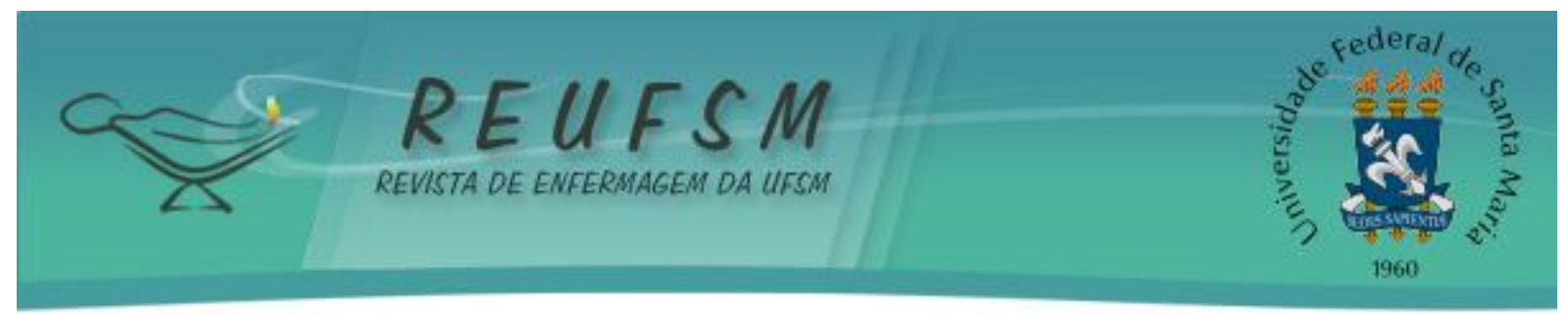

CONCLUSIÓN

El aspecto más satisfactorio de la Herramienta Web está relacionado con la percepción de ayuda que proporciona, debido probablemente a que los sucesivos usos de sus procedimientos permiten superar fácilmente dichas dificultades iníciales, aunque una de las limitaciones de este estudio estaba relacionada con la percepción de ayuda por parte de los alumnos que podría estar asociada con su nota en la asignatura. El uso de la Herramienta Web Nanda.es es altamente satisfactorio para los estudiantes de enfermería por la ayuda que obtienen en la realización de los planes de cuidados y diagnósticos.

La principal implicación de la herramienta está relacionada con la aplicabilidad del Proceso de Atención de Enfermería. El manejo de esta Herramienta ayuda al conocimiento y comprensión de la taxonomía NNN y a conseguir una mayor eficiencia en el proceso de atención en enfermería, que puede ser de gran utilidad para la aplicabilidad en la práctica clínica.

\section{BIBLIOGRAFÍA}

1. Diagnósticos enfermeros: definiciones y clasificación 2012-2014 (NANDA International). Madrid: Elsevier; 2012.

2. Lee MB, Brysiewicz P. Enhancing problem solving and nursing diagnosis in year III Bachelor of Nursing students. Nurse Educ Today. 2009;29(4):389-97.

3. Pérez Rivas FJ, Ochandorena Juanena M, Santamaría García JM, García López M, Solano Ramos V, Beamud Lagos $M$, et al. Aplicación de la metodología enfermera en atención primaria. Rev Calidad Asistencial. 2006;21(5):247-54.

4. Yönt GH, Khorshid L, Eser I. Examination of nursind diagnoses used by nursing students and their opinions about nursing diagnoses. Int J Nurs Terminol Classif. 2009;20(4):162-8.

5. Echevarría-Pérez $P$, Conesa $M$, Flores JA, Giménez $M$. Identificación de problemas de aprendizaje en taxonomía NANDA mediante la herramienta Web e-Nanda. VII Simposium Internacional de Diagnósticos de Enfermería; 2008 out 23-24; Barcelona. Barcelona: AENTDE; 2008. ISBN: 978-84-612-6839-9. p. 493-7.

6. Domingo Pozo M, Gómez Robles FJ, Torres Figueiras M, Gabaldón Bravo EM. Del cuidado registrado al diagnóstico enfermero. Enferm Clin. 2005;15(5):267-74.

7. Lunney M. Critical thinking and accuracy of nurses' diagnoses. Int J Nurs Terminol Classif. 2003;14(3):96-107.

8. Martínez Martínez C, Cañadas Bustos A, Rodríguez Paredes E, Tornel García S. Dificultades en la valoración enfermera. Enferm Clín. 2003;13(4):195-201.

9. Carpenito-Moyet LJ. Invited paper: teaching nursing diagnosis to increase uilization after graduation. Int J Nurs Terminol Classif. 2010;21(3):124-33.

10. Tesoro MG. Effects of using the developing nurses' thinking modelo on nursing students' diagnostic accuracy. J Nurs Educ. 2012;51(8):436-43.

11. Billings DM. Wikis and blogs: consider the possibilities for continuing nursing education. J Contin Educ Nurs. 2009;40(12):534-5.

12. Hansen M, Erdley S. YouTube and other Web 2.0 applications for nursing education. Online J Nurs Inform [Internet]. 2009 [acesso em 2013 out 23];13(3):1-20. Disponível em: http://ojni.org/13_3/Hansen_Erdley.pdf. 


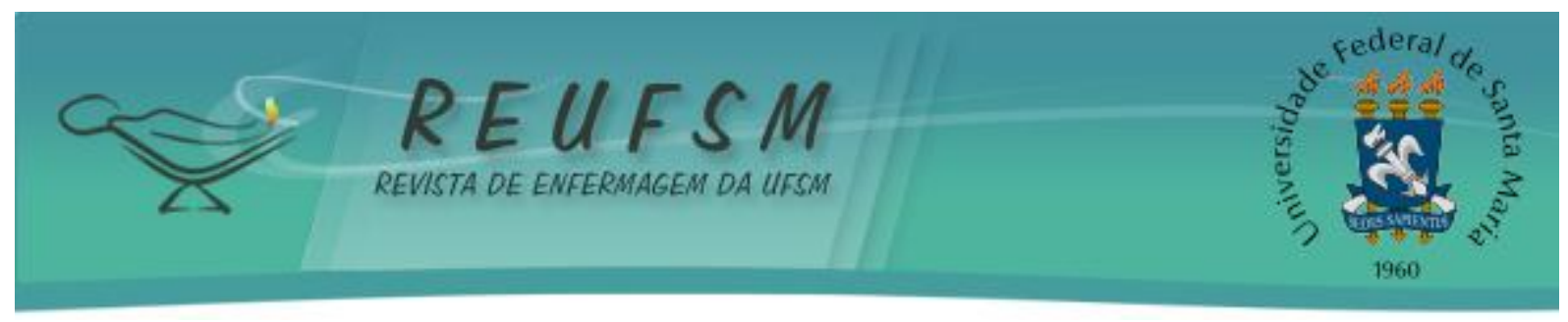

13. Billings DM. Meaningful use of Web 2.0 tools for teaching and learning. J Contin Educ Nurs. 2012;43(4):152-3.

14. Cavalcante RB, Otoni A, Bernardes MFVG, Cunha SGS, Santos CS, Silva PC. Experiências de sistematização da assistência de enfermagem no Brasil: um estudo bibliográfico. Rev Enferm UFSM [Internet]. 2011[acesso em 2013 out 23];1(3):461-71. Disponível em: http://cascavel.ufsm.br/revistas/ojs-2.2.2/index.php/reufsm/article/view/2832.

15. Román Cereto M, Campos Rico A, Viñas Heras C, Palop R, Zamudio Sánchez A, Domingo García R, et al. Las taxonomías enfermeras NANDA, NOC y NIC en la práctica asistencial hospitalaria. Enferm Clin. 2005;15(3):163-6.

16. Kurashima S, Kobayashi K, Toyabe S, Akazawa K. Accuracy and efficiency of computeraided nursing diagnosis. Int J Nurs Terminol Classif. 2008;19(3):95-101.

Data de recebimento: 19/09/2013

Data de aceite: 22/05/2014

Contato com autor responsável: Francisca Sánchez Ayllón

Endereço postal: Campus de Los Jerónimos S/N 30107 Guadalupe (Murcia). España.E-mail: fsayllon@ucam.edu 